\title{
Interações Planta-Inseto: Aspectos Biológicos e Ecológicos do Mutualismo Cecropia-Azteca
}

\author{
Gabriela Vasconcelos de Oliveira, Michele Martins Correa ${ }^{\bowtie}$ \&acques Hubert Delabie
}

Universidade Estadual do Sudoeste da Bahia, e-mail: oliveira.gvo@gmail.com, mcorrea@uesb.edu.br, jacques.delabie@gmail.com.

\section{EntomoBrasilis 8 (2): 85-9o (2015)}

Resumo. Uma associação bem conhecida dentre as interações ecológicas é o mutualismo entre plantas do gênero Cecropia e formigas, na maioria das vezes pertencentes ao gênero Azteca. Nesta interação, as formigas nidificam nas domáceas presentes no tronco oco das mirmecófitas e se alimentam de recursos alimentares fornecidos pela planta, conhecidos como corpúsculos mullerianos. Em compensação, as formigas protegem a planta hospedeira contra o ataque de herbívoros e a invasão por trepadeiras, e nutrem-na através de resíduos abandonados no tronco. Entretanto, a efetividade desta associação pode variar em função de vários fatores, como por exemplo, a espécie de formiga associada e o tipo de habitat da planta mirmecófita. Cerca de 10\% das espécies de Cecropia perderam suas características atrativas, como por exemplo, espécies que habitam ilhas e altas altitudes. Aspectos como a riqueza de formigas associadas e estudos moleculares evidenciam que a colonização destas plantas ocorreu diversas vezes e independentemente por alguns gêneros de formigas. A pressão de seleção exercida por algumas espécies de formigas pode ter sido um dos fatores decisivos para o desenvolvimento dos traços atrativos para as formigas em Cecropia. Com a evolução destas características as formigas puderam se tornar um parceiro efetivo da planta, facilitando a co-evolução de interações mais específicas.

Palavras-chave: Associação; Benefícios; Co-evolução; Formigas; Mirmecófita.

\section{Plant-Insect Interactions: Biological and Ecological Aspects of Cecropia-Azteca Mutualism}

Abstract. A well-known association is the mutualism between plants is between plant species of the genus Cecropia and ants, mostly belonging to the genus Azteca. In this association, the ants nest in domatia present in the hollow trunk of this myrmecophytes and feed on food bodies provided by the plant, known as mullerian bodies. In return, the ants protect the host plant against herbivore attacks and invasion by vines, and nourish it through waste laid in the trunk. However, the effectiveness of this association may vary with several factors, such as the associated ant species and the habitat type of the myrmecophyte. About $10 \%$ of Cecropia species lost their attractive characteristics, such as species living in high altitude and inhabit islands. Aspects like associated ant richness and molecular studies show that the colonization of these plants by ants occurred several times and independently by different genera. The selection pressure exerted by some species of ants may have been one of the decisive factors for the development of attractive traits for ants in Cecropia. With the evolution of these features ants might become an effective partner of the plant, facilitating the co-evolution of specific interactions.

Keywords: Ants; Association; Benefits; Co-evolution; Myrmecophyte.

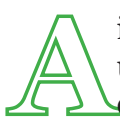
interação entre formigas e plantas é considerada como uma das relações ecológicas mais comuns, conspícuas e geograficamente difundida em todos os ecossistemas terrestres (DÁTTiLo et al. 2009). Essa interação tem-se mostrado importante para a defesa das plantas contra herbívoros e, em muitos casos, para a reprodução das espécies envolvidas (BEATTIE 1985; HöLLDOBLER \& WILSON 1990; HeIL \& MCKEY 2003; RiCO-GRAY \& OliveIRA 2007). Algumas formigas se alimentam de nectários florais e extraflorais, agindo raramente como polinizadoras (Schultz \& McGlynn 2000; Heil \& Mckey 2003). Alimentamse também do elaiossomo, tecido que cobre as sementes de algumas plantas, atuando, consequentemente, como dispersoras das mesmas (BeAtTie 1985; HölLDOBler \& Wilson 1990; Jolivet 1996; Delabie et al. 2003). Em interações mais específicas, algumas plantas formam estruturas onde as formigas constroem seu ninho, chamadas "domáceas", que têm a forma de espinhos inchados, raízes, bulbos, tubérculos, caules ocos ou bolsas laminares formadas na base das folhas (JANZEN 1969; JoLIVET 1996; Hölldobler \& Wilson 1990; Delabie et al. 2003; Heil \& MCKey 2003; Rico-Gray \& Oliveira 2007). Plantas também podem produzir néctar ou corpos alimentares ricos em lipídio e/ou proteína "em troca” da proteção contra outros artrópodes herbívoros (Hölldobler \& Wilson 1990; Schultz \& McGlynN 2000; Delabie et al. 2003).
Exemplos bem conhecidos do mutualismo plantas-formigas são as associações entre árvores do gênero Macaranga (Euphorbiaceae) e formigas do gênero Crematogaster e Camponotus no sudeste da Ásia (FIAla et al. 1991; Fiala et al. 1999), e plantas do gênero Acacia (Mimosoideae, Fabaceae) e formigas do gênero Pseudomyrmex na América Central (JANZEN 1966). Nestas associações, as plantas hospedeiras desenvolvem domáceas onde as formigas nidificam e elas produzem corpos alimentares dos quais as formigas se alimentam (Beattie 1985; Jolivet 1990; Fiala et al. 1991; Fiala et al. 1999; Rico-Gray \& Oliveira 2007). Em compensação, as formigas mantêm a planta livre de herbívoros e de plantas competidoras, tais como trepadeiras (JANZEN 1966, 1969; FiALA et al. 1991; Fiala et al. 1999; Rico-Gray \& Oliveira 2007).

A análoga neotropical de Macaranga são as árvores do gênero Cecropia (Urticaceae), associadas comumente a formigas do gênero Azteca (Fiala et al. 1991; Fiala et al. 1999). Nesta associação, assim como naquelas descritas anteriormente, a Cecropia hospedeira oferece local de nidificação e corpos alimentares às formigas e, em contrapartida, estas oferecem à planta proteção contra herbívoros e plantas trepadeiras (JANZEN 1969, 1973; Schupp 1986; Jolivet 1990; Davidson \& Fisher 1991; VASCONCELOS \& CASIMIRO 1997). Neste contexto, esta revisão tem como objetivo apresentar de modo sintético as características 
biológicas e ecológicas da interação entre Cecropia e Azteca.

\section{CECROPIA E SEU PAPEL NA INTERAÇÃO}

Plantas do gênero Cecropia são árvores pioneiras de crescimento rápido e exclusivamente neotropicais (SCHUPP 1986; BERG \& ROSSELLI 2005), bastante utilizadas em reflorestamentos de áreas degradadas (LORENZI 2002). Este gênero possui 61 espécies (BERG \& Rosselli 2005; Gaglioti \& Romaniuc 2012) que se distribuem desde o sul do México até o norte da Argentina (DAvidson 2005). No Brasil, existe cerca de 20 espécies, principalmente na região Norte (GAGLioti \& Romaniuc 2012).

Cerca de $80 \%$ das espécies de Cecropia são mirmecófilas (DAvidson 2005), ou seja, possuem estruturas morfológicas que fornecem abrigo às formigas (DAvidson \& MCKEY 1993; JoLIVET 1996; DeLABIE et al. 2003; HeIL \& MCKey 2003), com uma taxa de ocupação elevada (Longino 1991). Mirmecófitas evoluíram tanto para fornecer recursos alimentares para as formigas, quanto para conceder locais de nidificação dentro de partes modificadas de seu corpo ou em estruturas pré-adaptadas (DAvidson 2005). As mirmecófitas do gênero Cecropia possuem o tronco oco e segmentado formando câmeras internas (DUMPERT 1978) onde as formigas nidificam (JANZEN 1969). A parede do tronco possui locais menos robustos conhecidos como prostoma, localizados nos entrenós e que não possuem tecido vascularizado, por onde as rainhas penetram em uma das câmeras sem romper os tecidos vasculares da planta (WheEler 1942; Dumpert 1978; DAvidson \& Fisher 1991; DAvidSON 2005). Além do lugar para nidificação, Cecropia também oferece recursos alimentares às formigas, uma vez que produz corpúsculos müllerianos em uma estrutura aveludada localizada na base do pecíolo (triquílio), os quais são ricos em glicogênio (JANZEN 1969; DUMPERT 1978). Algumas espécies de Cecropia também possuem corpúsculos em pérolas ("pearl bodies"), presentes como tricomas na superfície inferior da folha, contendo proteína e ricos em lipídios (RICKSON 1977), dos quais as formigas alimentam-se.

A maioria das Cecropia ocorre em habitats abertos, beira de estradas e clareiras de vegetação secundária (HARADA 1982; LoNGINO 1989a; BERG \& RosselLi 2005), porém algumas espécies podem ser encontradas em florestas maduras e primárias, lugares inundados, matas úmidas e matas ciliares (HARADA 1982; GUERRERO et al. 2010). Essas diferenças na ocupação de habitats refletem diferenças no crescimento da planta e na taxa de produção de recursos alimentares usados pelas formigas (DAvidson \& Fisher 1991). Espécies de Cecropia de ambientes abertos, como Cecropia concolor Willdenow, Cecropia obtusifolia Bertoloni, Cecropia pachystachya Trécul e Cecropia peltata Linnaeus, exigem mais luminosidade e possuem crescimento mais rápido de que aquelas mais tolerantes à sombra, apresentando também folhas de vida curta (DAVIDSON \& MCKey 1993; Berg \& Rosselli 2005). Já as espécies mais tolerantes à sombra, como Cecropia distachya Huber, Cecropia insignis Liebmann e Cecropia purpurascens Berg, possuem crescimento lento e folhas de vida longa e, por isso, produzem prostomas e triquílios mais cedo durante o seu desenvolvimento (LONGINo 1989a; DAvidSON \& MCKEY 1993; BERG \& Rosselli 2005). A maior produção de corpos müllerianos nas plantas tolerantes à sombra é justificada pela maior necessidade destas plantas em manterem suas folhas, assim, quanto maior a produção, mais formigas estarão presentes neste local, aumentando a eficácia da proteção (Folgarait \& DAVIDSON 1994). Apesar de produzirem triquílios mais tarde, as espécies de ambientes abertos fornecem mais recursos às formigas ao longo da vida da planta, em razão do número de folhas que produzem ao longo da vida ser maior (DAVIDSON \& MCKEY 1993).

Cerca de 10\% das espécies de Cecropia não possuem estruturas que abrigam as formigas, ou possuem, mas não abrigam porque perderam suas características atrativas (triquílio e corpúsculos mullerianos) (Jolivet 1990), como espécies que habitam ilhas e altas altitudes - acima de 2000 m (JANZEN 1973; RICKSON 1977;
Jolivet 1990). Por sua vez, as espécies brasileiras Cecropia sciadophylla Martius e Cecropia hololeuca Miquel não possuem triquílio e não formam corpúsculos mullerianos (JOLIVET 1990). De acordo com JANZEN (1973) e Rickson (1977), as populações de Cecropia de ilhas e de altitudes elevadas enfrentam pressão por herbivoria e invasão por trepadeiras consideravelmente reduzidas, e, por isso, podem ter perdido suas características atrativas às formigas como uma forma de conservar sua energia $\mathrm{e}$ redirecioná-la para aumentar seu crescimento e/ou fecundidade. Entretanto, a ausência de formigas em Cecropia de altitudes elevadas pode variar entre diferentes áreas, de acordo com a umidade do local, uma vez que foi registrada a ocorrência de formigas habitando uma espécie de Cecropia a mais de $2.000 \mathrm{~m}$ de altitude na Colômbia (JANZEN 1973).

Plantas introduzidas fora do seu habitat natural de ocorrência também podem perder suas características atrativas. A espécie C. peltata foi introduzida experimentalmente na Malásia, onde não é colonizada por formigas nativas, e apresenta uma perda progressiva das características mirmecófitas (Jolivet 1996). Quando desenvolve corpúsculos mullerianos, estes não são removidos por nenhum organismo nativo (PUTZ\& HolBROOK 1988). Plantas em que os corpúsculos mullerianos não são removidos apresentam produção menor quando comparado à produção de plantas em que os corpúsculos são removidos (Johnson et al. 1992). Assim, é provável que a produção de corpos alimentares seja estimulada pela ocorrência das próprias formigas na planta (JOLIVET 1996).

\section{ASSOCIAÇÃO DE CECROPIA COM FORMIGAS}

Na Região Neotropical, várias espécies de formigas podem habitar as plantas de Cecropia, entretanto a associação mais comum é com o gênero Azteca. Outros dois gêneros com espécies frequentes que habitam e conseguem manter ninhos maduros e persistentes em Cecropia são Pachycondyla e Camponotus (Davidson \& Fisher 1991; Longino 1991; YU \& DAvidson 1997). Além destas, vários gêneros de formigas podem residir ocasionalmente em Cecropia (Longino 1989a), ocupando partes inferiores do tronco e forrageando fora da planta como Cephalotes, Crematogaster, Pheidole, Pseudomyrmex, Solenopsis e Wasmannia (DAvidson \& FISHER 1991; LONGINO 1991).

Apesar do mutualismo Cecropia e Azteca ser muito comum, nenhuma espécie de Azteca é moradora estrita de uma única planta hospedeira (Longino 1989a). Cada espécie de Cecropia pode ser habitada por mais de uma espécie de formiga, enquanto as Azteca, por sua vez, habitam mais de uma espécie de Cecropia, sendo que algumas habitam até plantas de outros gêneros como Cordia, Tachigali, Tococa e Triplaris (Longino 1989a; LongIno 1991; DAvidson \& Mckey 1993; Longino 1996). Apenas algumas espécies de formigas são habitantes obrigatórias de mirmecófitas, tais como formigas do gênero Crematogaster que procuram por qualquer tipo de cavidade disponível, enquanto que as Azteca, frequentemente, nidificam em plantas adaptadas (JoLIVET 1996). As espécies do gênero Azteca apresentam diferentes graus de especificidade com a planta hospedeira (LongINo 1991). Formigas deste gênero são conhecidas por se associar com mirmecófitas de pelo menos 16 gêneros e nove famílias (DAVIDSON \& MCKEY 1993).

De acordo com Longino (1989a), a associação entre Azteca e Cecropia é mais comum devido ao tamanho e forma das colônias destas formigas. Enquanto a maioria das formigas arborícolas forma pequenas colônias, ou mesmo grandes colônias distribuídas num espaço reduzido, ocupando apenas uma parte do tronco da planta, as colônias de Azteca são capazes de se expandir até ocupar todo volume da árvore, onde podem eliminar colônias da mesma ou de outras espécies já ocorrendo ali. Entretanto espécies como Camponotus, Crematogaster, Pachycondyla e Solenopsis podem ser encontradas em árvores que abrigam colônias maduras de Azteca, assim como colônias de mais de uma espécie de Azteca podem ocorrer simultaneamente na mesma planta (HARADA \& 


\section{AZTECA E SEU PAPEL NA INTERAÇÃO}

Azteca é um gênero de formigas exclusivamente neotropical, contendo 84 espécies (BolTon 2013) distribuídas desde o México até Paraguai e Argentina (WheELer 1942; HARADA 1982). Todas as espécies são arborícolas, mas podem apresentar hábitos de nidificação e habitats diferentes. Algumas nidificam em galhos secos ou mortos, outras em troncos vivos, ou ainda em pequenas dilatações do caule ou das folhas, algumas constroem ninhos de cartão dentro do tronco enquanto outras constroem ninhos de cartão fora dele (HARADA 1982; Longino 1989b; Longino 1991; GuERrERo et al. 2010).

Dentro do gênero Azteca, 21 espécies apresentam mutualismo com Cecropia, sendo que a maioria também pode habitar outras mirmecófitas (HARADA \& BENSON 1988). Destas, 12 espécies foram registradas no Brasil e nove são consideradas especialistas de Cecropia (HARAdA \& BENSON 1988). Dentre elas Azteca alfari Emery é a que possui distribuição mais ampla, ocorrendo desde o México até a Argentina (HARAdA \& BENSON 1988; Longino 1991) e nidifica em qualquer espécie de Cecropia mirmecófita, principalmente em áreas de capoeira e áreas abertas como pastos e beira de estrada (HARADA 1982; HARADA \& BENSON 1988; LONGINO 1989b; Longino 1991). Já Azteca constructor Emery e Azteca xanthochroa Roger são mais comuns em florestas fechadas (LoNGINo 1991). A partição entre espécies de Azteca pode variar de acordo com a altitude; por exemplo, na Costa Rica, $A$. alfari é mais comum em baixas altitudes, enquanto que $A$. constructor é mais frequente em áreas mais elevadas (LoNGINo 1989a).

Numa outra relação mutualística, as espécies do gênero Azteca também são conhecidas por criarem populações de insetos hemípteros sugadores (Coccoidea) dentro dos troncos ocos de Cecropia, os quais proveem às formigas açúcar, vitaminas e aminoácidos em abundância, que usam para a alimentação da colônia (CARrol 1983; Delabie 2001; JohnSON et al. 2001). Esses insetos, em compensação, são protegidos de seus inimigos naturais pelas formigas e por estarem instalados em sites mais favoráveis a sua tomada alimentar (Ness et al. 2009). Com a abundância de carboidratos fornecidos pelos hemípteros, a dieta da colônia de formigas está provida por esses nutrientes em excesso quando comparados às proteínas fornecidas pelos corpúsculos da planta. O crescimento da colônia se torna então limitado principalmente pela quantidade de proteínas disponível. Assim, os carboidratos são gastos em comportamentos agressivos, dominando e protegendo o território onde as proteínas se encontram (os triquílios) e são essenciais para o crescimento da colônia (DAVIDSON \& MCKEY 1993). A capacidade de nidificar próximo de algum recurso alimentar facilmente acessível reduz os custos de forrageamento (Ness et al. 2009), contribuindo para intensificação do comportamento agressivo e a dominância da colônia (DAVIDSON \& MCKEY 1993).

Além de proteger os triquílios, a presença das formigas beneficia a planta hospedeira de outras formas, uma vez que as Azteca removem trepadeiras e outras plantas que competem com a hospedeira por luz e nutrientes, influenciando positivamente a reprodução e sobrevivência da Cecropia (JANZEN 1969; SchupP 1986). Embora a remoção de trepadeiras traga vários benefícios para a planta, DAVIDSON \& MCKEY (1993) sugerem que a principal vantagem para as formigas em remover outras plantas é a redução da taxa de invasão por outras formigas dominantes que poderiam usar as trepadeiras como ponte.

A planta hospedeira também é beneficiada pelos nutrientes fornecidos pelas formigas através dos resíduos depositados nos entrenós dentro do tronco (SAGERS et al. 2000; DeJEAN et al. 2012). Cerca de $93 \%$ do nitrogênio assimilado pelas plantas de Cecropia é derivado das formigas ou dos depósitos de resíduos das formigas (SAGERs et al. 2000). De acordo com estes autores, plantas ocupadas por Azteca assimilam mais nitrogênio em comparação com aquelas não ocupadas pelas formigas. A maior assimilação de nitrogênio pode conferir uma grande vantagem para as plantas que abrigam Azteca, uma vez que o nitrogênio pode limitar o crescimento nesta espécie pioneira.

Além destes benefícios, a ocorrência da Azteca na planta reduz o número de herbívoros, tais como formigas cortadeiras e insetos mastigadores, assim como os danos causados por eles (Schupp 1986; Jolivet 1990; Vasconcelos \& CASIMIRo 1997), influenciando positivamente o crescimento das árvores que habitam (Schupp 1986; VASCONCELOS \& CASIMIRo 1997). No Equador, a densidade de herbívoros e a taxa de herbivoria foram menores em indivíduos de Cecropia habitados por formigas do que em plantas que não abrigavam formigas, tanto naturalmente quanto removidas experimentalmente (Schupp 1986). Nesta área, A. constructor foi eficaz em defender sua hospedeira contra herbivoria foliar por insetos mastigadores, e consequentemente os indivíduos defendidos pelas formigas cresceram significativamente mais rápido do que aqueles sem formigas. Segundo VASCONCELOS \& CAsımiro (1997), plantas de Cecropia não ocupadas por A. alfari foram atacadas com maior frequência pelas formigas cortadeiras e tiveram maior taxa de mortalidade que aquelas ocupadas. Além disso, segundo os mesmos autores, as espécies de Cecropia ocupadas com menor frequência por Azteca foram mais atacadas por formigas cortadeiras, mesmo sendo menos palatáveis para elas. A eficácia da defesa é resultado da mobilidade e da relativa baixa especificidade das formigas, já que podem ser efetivas contra vários tipos de insetos (HeIL et al. 2001). Entretanto a efetividade das formigas na defesa contra herbívoros pode variar em função da espécie de formiga mutualística e agressividade da mesma (DAvidson \& MCKey 1993; BRonstein 1998; Bruna et al. 2004).

Embora diversos estudos demonstrem associação positiva nesta interação, ainda hoje não existe consenso se Azteca protege efetivamente ou não as árvores de Cecropia contra os herbívoros (FÁVERI \& VASCONCELOS 2004). Alguns autores mostraram que a ocorrência de Azteca não influencia a taxa de herbivoria sofrida pela planta, apontando que plantas associadas à Azteca sofrem a mesma taxa de herbivoria que aquelas desprovidas destas formigas (ANDrade \& Carauta 1982; Putz \& Holbrook 1988; FÁVERI \& VASCONCELOS 2004). No entanto, nesses estudos, as taxas de herbivoria foram baixas, provavelmente devido à baixa ocorrência de herbívoros ou à concentração elevada de compostos secundários nas folhas, permitindo aos indivíduos de Cecropia sobreviverem na ausência das formigas (FÁvERI \& VASCONCELOS 2004). Em Cecropia, a concentração de taninos e compostos fenólicos varia entre as espécies e é maior quando a planta cresce em pleno sol e recebe grande intensidade de luz (Folgarait \& DAVIDSON 1994). Assim, a eficiência de proteção contra herbívoros varia com a espécie de Azteca associada e com as características do meio em que a planta vive, tais como abundância de herbívoros e trepadeiras (Putz \& Holbrook 1988; Bronstein 1998; FÁveri \& VASCONCELOS 2004). Varia também em função da incidência solar, que influencia diretamente a produção de compostos secundários (FÁVERI \& VASCONCELOS 2004). O sucesso das plantas de Cecropia em áreas com baixa abundância de herbívoros e trepadeiras demonstra que este gênero não depende obrigatoriamente da associação mutualística com Azteca para sobreviver (Putz \& HOLBROOK 1988; WETTERER 1997).

\section{ASPECTOS CO-EVOLUTIVOS DA INTERAÇÃO ENTRE CECROPIA E AZTECA}

A riqueza e diversidade de formigas associadas à Cecropia indicam a frequência e facilidade em que o mutualismo evoluiu ao longo do tempo, e fornecem evidências de que Cecropia foi colonizada diversas vezes e independentemente por alguns gêneros de formigas (DAVIDSON \& MCKEY 1993). Estudos moleculares mostram que as espécies de Azteca associadas com Cecropia não formam um grupo monofilético, indicando mais uma vez processos 
T independentes de colonização destas plantas (Ayala et al. 1996). Em árvores como Cecropia, troncos largos, ocos e com medulas fracas provavelmente evoluíram como uma forma de minimizar o custo metabólico do tecido arbóreo que suporta suas grandes folhas (WHITE 1983; DAvidson \& MCKEY 1993). Uma vez evoluídos como domáceas, tais troncos formaram lugares de nidificação acessíveis para formigas de tamanhos corporais diversos. Para plantas com esta estrutura, a proteção por formigas pode ser vantajosa porque os meristemas são bastante suscetíveis a danos por insetos. Como a maioria destas plantas produz um único ou poucos meristemas, o custo de perdê-lo(s) pode ser bem elevado (DAVIDSON \& MCKEY 1993).

Para muitas espécies de formigas de dietas generalizadas, lugares de nidificação seguros podem ser mais limitantes do que os recursos alimentares. Assim, formigas que ocuparam o tronco de Cecropia podem ter beneficiado de várias formas a planta hospedeira e exercido pressão de seleção, podendo ter sido um dos fatores decisivos para o desenvolvimento de outros traços atrativos para as formigas, tais como prostoma e o triquílio com corpos müllerianos (DUMPERT 1978; DAVIDSON 2005). O fornecimento de recursos alimentares garantiu que as formigas pudessem se tornar um grupo de insetos parceiro efetivo da planta, e facilitou a evolução de interações mais específicas (DAVIDSON \& MCKEY 1993). Assim, uma vez que estas características evoluíram, elas podem ter sido modificadas pela pressão de seleção, exercitada por espécies de formigas mais especializadas (DAVIDSON \& FisHER 1991).

Entre as formigas associadas obrigatoriamente à Cecropia, ou seja, aquelas que utilizam a própria estrutura da planta como local de nidificação e apresentam adaptações do seu comportamento e estruturas morfológicas adequadas para esse tipo de vida (HARADA 1982), o reconhecimento e uso de prostoma e corpos müllerianos pelas rainhas e operárias podem ser considerados traços coadaptados derivando da associação com Cecropia. Além disso, muitos aspectos da defesa da planta são restritos a essas associações, tais como a remoção de trepadeiras e herbívoros, uma vez que é necessário que as formigas distingam a hospedeira das outras plantas (HeIl et al. 2001). Já formigas que não são obrigatoriamente associadas à Cecropia, e que forrageiam ou habitam a planta de modo oportuno, tendem a não explorar os corpos müllerianos como recurso alimentar (PuTz \& Holbrook 1988; WetTERER 1997; DAVIDSON \& Fisher 1991), nem o prostoma como entrada para uma cavidade dentro do tronco oco (Longino 1989a). Outro traço coadaptativo é o formato da cabeça das rainhas de $A$. alfari (espécie de associação obrigatória), que parecem ter evoluído para facilitar sua entrada perfurando o prostoma do tronco de Cecropia (Longino 1989a). Além disso, as rainhas dessa espécie parecem ser especialmente bem adaptadas para ocupar indivíduos de Cecropia, pois possuem sensibilidade olfativa aguçada para localizar essas plantas hospedeiras (LoNGINO 1989a).

\section{CUSTO DA INTERAÇÃO}

A localização dos corpúsculos müllerianos na base dos pecíolos das folhas de Cecropia atrai as operárias das formigas residentes principalmente para este local e troncos adjacentes (DAVIDSON 2005). Formigas cortadeiras e outros insetos como gorgulhos (Curculionidae) e besouros do gênero Coelomera (Chrysomelidae) são comuns em troncos de Cecropia (JoliveT 1990), sendo que as formigas cortadeiras podem danificar ou destruir os meristemas apicais das Cecropia jovens e remover folhas inteiras cortando seus pecíolos (VASCONCELOS \& CASIMIRO 1997). O principal impacto positivo deste mutualismo entre Cecropia e formigas residentes como Azteca é provavelmente a proteção dos pecíolos, que suportam folhas grandes e inteiras, pois o corte ou enfraquecimento dele pode ser desastroso para plantas que investem muitos recursos em grandes folhas inteiras, como é o caso de Cecropia (DAVIDSON 2005).
A herbivoria parece ser um fator importante na indução do recrutamento das formigas e para a defesa da planta hospedeira (Agrawal \& Dubin-Thaler 1999; Bruna et al. 2004), uma vez que as formigas respondem aos danos causados nas folhas (AGRAWAL 1998). Ao serem cortadas, as folhas liberam compostos voláteis, que podem servir como sinal de que herbívoros estão ali presentes, e então operárias de Azteca respondem a estas substâncias recrutando outros indivíduos, aumentando a ocorrência de formigas na folha cortada (Agrawal \& Dubin-Thaler 1999). Para Agrawal (1998), as formigas atuam com eficácia como defesa induzida das plantas por causa da sua mobilidade e capacidade de se organizar e responder rapidamente. Entretanto, a resposta pode variar com a espécie de formiga associada à planta e com o auxílio de feromônios de alarme soltos pelas formigas, e que aceleram a resposta de defesa à herbivoria (BRuna et al. 2004).

A manutenção da defesa biótica por parte da planta gera um custo elevado, e é mantida porque os benefícios, no caso a redução da herbivoria, excedem os gastos. A produção de recursos alimentares deve ser incrementada constantemente e possui maiores custos de manutenção do que as defesas químicas (DAvidson \& FisheR 1991), alocando para a defesa, recursos que seriam dirigidos ao crescimento ou reprodução da planta (Gianoli et al. 2008). Assim, os investimentos em defesa devem ser diretamente associados ao valor do tecido da planta e à probabilidade de ataques por herbívoros (GIANOLi et al. 2008). Defesas químicas podem ter sido reduzidas ou perdidas em algumas plantas mirmecófitas (JANZEN 1966), e as formigas cumprem as funções que seriam fornecidas por essas defesas químicas (HeIL et al. 2001). Outras mirmecófitas produzem defesas químicas no seu desenvolvimento inicial, quando ainda não possuem características atrativas às formigas e, consequentemente, não possuem defesa biótica (ColeY 1986; DAvidson \& Fisher 1991). Entretanto VAL \& Dirzo (2003) mostraram que, mesmo depois de colonizadas pelas formigas, algumas espécies de Cecropia produzem mais defesas químicas e físicas que quando jovens e não colonizadas, auxiliando na defesa contra diferentes herbívoros. Já as espécies ocupadas por formigas não especializadas (oportunistas que não defendem a planta) possuem menor quantidade de triquílios ativos e investem mais em defesas químicas, com, por exemplo, maior concentração de taninos em suas folhas (DAVIDSON \& FISHER 1991). O custo elevado da defesa biótica em Cecropia é mais evidente quando as características atrativas para as formigas são perdidas pela ausência da seleção imposta pelas formigas associadas às plantas (Rickson 1977; Putz \& Holbrook 1988; Johnson et al. 1992; DAVISON 2005).

\section{CONSIDERAÇÕES FINAIS}

Esta revisão aborda os principais aspectos biológicos e ecológicos do mutualismo entre Cecropia e Azteca, que é bastante comum nos ecossistemas e indica fortes evidências evolutivas nesta interação, tendo suporte nos mecanismos de abrigo e/ou alimento da Cecropia que atraem formigas que as protejam contra herbívoros e trepadeiras (DUMPERT 1978; DAvidson \& MCKEY 1993; HeIl et al. 2001; DAVIDSON 2005). A manutenção da defesa feita pelas formigas gera um custo elevado para a planta, e é mantida porque os benefícios, no caso a redução da herbivoria, excedem os gastos, no caso a produção de recursos alimentares (DAVIDSON \& FISHER 1991)

Sabe-se que parceiros mutualistas e antagonistas exercem pressões seletivas diferentes no hospedeiro, e essa diferença pode se refletir nas características fenotípicas da planta (DUMPERT 1978; Davidson \& MCKeY 1993; Heil et al. 2001; DavidSON 2005). Contudo, estudos com esse enfoque ainda são escassos na literatura (e.g. HeIL et al. 2001; VAL \& Dirzo 2003; BRUNA et al. 2004; FÁveri \& VASCONCELOS 2004). Neste contexto, trabalhos futuros poderiam focar seus objetivos no esclarecimento de como a biologia das formigas mutualistas Azteca impactam o desenvolvimento das diferentes espécies de Cecropia e como essa interação é afetada pelas características bióticas e abióticas 
do habitat das espécies.

\section{REFERÊNCIAS}

Agrawal, A.A. \& B.J. Dubin-Thaler, 1999. Induced responses to herbivory in the Neotropical ant-plant association between Azteca ants and Cecropia trees: response of ants to potential inducing cues. Behavioral Ecology and Sociobiology, 45: 4754 .

Agrawal, A.A., 1998. Leaf damage and associated cues induce aggressive ant recruitment in a Neotropical ant-plant. Ecology, 79: 2100-2112.

Andrade, J.C. \& J.P.P. Carauta, 1982. The Cecropia-Azteca association: a case of mutualism? Biotropica, 14: 15 .

Ayala, F.J.; J.K. Wetterer, J. Longino \& D.L. Hartl, 1996. Molecular phylogeny of Azteca ants (Hymenoptera: Formicidae) and the colonization of Cecropia trees. Molecular Phylogenetics and Evolution, 5: 423-428.

Beattie, A.J., 1985. The evolutionary ecology of ant-plant mutualisms. Cambridge, Cambridge University Press, 182p.

Berg, C.C. \& P.F. Rosselli, 2005. Flora Neotropica Monograph 94: Cecropia. New York, Organization for Flora Neotropica, 230p.

Bolton, B., 2013. New general catalogue of the ants of the world. Disponível em: <http://www.antwiki.org/wiki/images/c/c4/ NGC January 2013.pdf >. [Acesso em 01.01.2013].

Bronstein, J.L., 1998. The Contribution of Ant-Plant Protection Studies to Our Understanding of Mutualism. Biotropica, 30: 150-161.

Bruna, E.M., D.M. Lapola \& H.L. Vasconcelos, 2004. Interspecific variation in the defensive responses of obligate plant-ants: experimental tests and consequences for herbivory. Oecologia, 138: 558-565.

Carrol, C.R., 1983. Azteca (hormiga Azteca, Azteca ants, Cecropia ants), p. 691-693. In: Janzen, D.H. (Ed.) Costa Rican Natural History. Chicago, University of Chicago Press, 823p.

Coley, P.D., 1986. Costs and benefits of defense by tannins in a Neotropical tree. Ecology, 70: 238-241.

Dáttilo, W.; E.C. Marques, J.C.F. Falcão, D.D.O. Moreira, 2009. Interações mutualísticas entre formigas e plantas. EntomoBrasilis, 2: 32-36.

Davidson, D.W. \& B.L. Fisher, 1991. Symbiosis of ants with Cecropia as a function of light regime, p. 289-309. In: Huxley, C.R. \& D.F. Cutler (Eds.). Ant-Plant Interactions. New York, Oxford University Press, 601p.

Davidson, D.W. \& D. Mckey, 1993. The evolutionary ecology of symbiotic ant-plant relationships. Journal of Hymenoptera Research, 2: 13-83.

Davidson, D.W., 2005. Cecropia and its biotic defenses, p. 214-226. In: Berg, C.C. \& P.F. Rosselli. Flora Neotropica Monograph 94: Cecropia. New York, Organization for Flora Neotropica, 23op.

Dejean, A., F. Petitclerc, O. Roux, J. Orivel \& C. Leroy, 2012. Does exogenic food benefit both partners in an ant-plant mutualism? The case of Cecropia obtusa and its guest Azteca plant-ants. Comptes Rendus Biologies, 335: 214-219.

Delabie, J.H.C., 2001. Trophobiosis between Formicidae and Hemiptera (Sternorrhyncha and Auchenorrhyncha): an overview. Neotropical Entomology, 30: 501-516.

Delabie, J.H.C., M. Ospina \& G. Zabala, 2003. Relaciones entre hormigas y plantas: una introducción, p. 167-180. In: Fernández, F. (Ed.). Introducción a las hormigas de la región Neotropical. Bogotá, Acta Noturna, 398p.

Dumpert, K., 1978. The Social Biology of Ants. London, Pitman Publishing Limited, 289p.

Fáveri, S.B. \& H.L. Vasconcelos, 2004. The Azteca-Cecropia association: are ants always necessary for their host plants? Biotropica, 36: 641-646.

Fiala, B., A. Jakob, U. Maschwitz \& K.E. Linsenmair, 1999. Diversity, evolutionary specialization and geographic distribution of a mutualistic ant-plant complex: Macaranga and Crematogaster in South East Asia. Biological Journal of the Linnean Society, 66: 305-331.

Fiala, B., U. Maschwitz \& T.Y. Pong, 1991. The association between Macaranga trees and ants in South-east Asia, p. 263-270. In: Huxley, C.R. \& D.F. Cutler (Eds.). Ant-Plant Interactions. New York, Oxford University Press, 601p.

Folgarait, P.J. \& D.W. Davidson, 1994. Antiherbivore defenses of myrmecophytic Cecropia under different light regimes. Oikos, 71: 305-320.

Gaglioti, A.L. \& S.N. Romaniuc, 2012. Urticaceae, p. 331-361. In: Wanderley, M.G.L., G.J. Shepherd, T.S. Melhem, A.M. Giulietti \& Martins, S.E. (Eds.) Flora Fanerogâmica do Estado de São Paulo. Volume 7. São Paulo, FAPESP, 361p.

Gianoli, E., S. Sendoya, F. Vargas, P. Mejía, R. Jaffé, M. Rodríguez \& A. Gutiérrez, 2008. Patterns of Azteca ants' defence of Cecropia trees in a tropical rainforest: support for optimal defense theory. Ecological Research, 23: 905-908.

Guerrero, R.J., J.H.C. Delabie \& A. Dejean, 2010. Taxonomic contribution to the Aurita group of the ant genus Azteca (Formicidae: Dolichoderinae). Journal of Hymenoptera Research: Festschrift Honoring Roy Snelling, 19: 51-65.

Harada, A.Y. \& W.W. Benson, 1988. Espécies de Azteca (Hymenoptera, Formicidae) especializadas em Cecropia spp. (Moraceae): distribuição geográfica e considerações ecológicas. Revista Brasileira de Entomologia, 32: 423-435.

Harada, A.Y., 1982. Contribuição ao conhecimento do gênero: Azteca Forel, 1878 (Hymenoptera: Formicidae) e Aspectos da interação com plantas do gênero Cecropia Loefling, 1758. Dissertação (Mestrado em Entomologia) - Instituto Nacional de Pesquisas da Amazonia e Universidade do Amazonas. 181p.

Heil, M. \& D. Mckey, 2003. Protective Ant-plant Interactions as Model Systems in Ecological and Evolutionary Research. Annual Review of Ecology, Evolution, and Systematics, 34: 425-453.

Heil, M., B. Fiala, U. Maschwitz \& K.E. Linsenmair, 2001. On benefits of indirect defence: short- and long-term studies of antiherbivore protection via mutualistic ants. Oecologia, 126: 395-403.

Hölldobler, B. \& E.O. Wilson, 1990. The ants. Berlin, SpringerVerlag, $732 \mathrm{p}$.

Janzen, D.H., 1966. Coevolution of mutualism between ants and acacias in Central America. Evolution, 20: 249-275.

Janzen, D.H., 1969. Allelopathy by myrmecophytes: the ant Azteca as an allelopathic agent of Cecropia. Ecology, 50: 147153 .

Janzen, D.H., 1973. Dissolution of mutualism between Cecropia and its Azteca ants. Biotropica, 5: 15-28.

Johnson, C., D. Agosti, J.H. Delabie, K. Dumpert, D.J. Williams, M. Von Tschirnhaus \& U. Maschwitz, 2001. Acropyga and Azteca ants (Hymenoptera: Formicidae) with scale insects (Sternorrhyncha: Coccoidea): 20 million years of intimate symbiosis. American Museum Novitates, 3335, 18p.

Johnson, H.L., D. W. Davidson \& P. Folgarait, 1992. Experimental manipulation of food bodies in Cecropia. Proceedings-NCUR VI: $1542-1547$.

Jolivet, P., 1990. Relative protection of Cecropia trees against leaf-cutting ants in tropical America, p. 251-254. In: Meer, R.K.V., K. Jaffe, A. Cedeno (Eds.) Applied Myrmecology: A World Perspective. San Francisco, Westview Press, 741p.

Jolivet, P., 1996. Ants and Plants: an Example of Coevolution. Leiden, Backhuys Publishers, 303p.

Longino, J.T., 1989a. Geographic variation and community structure in an ant-plant mutualism: Azteca and Cecropia in Costa Rica. Biotropica, 21: 126-132.

Longino, J.T., 1989b. Taxonomy of the Cecropia-inhabiting ants in the Azteca alfari species group (Hymenoptera: Formicidae): evidence for two broadly sympatric species. Contributions in Science, Natural History Museum of Los Angeles County, 412: 1-16.

Longino, J.T., 1991. Azteca ants in Cecropia trees: taxonomy, 
colony structure, and behavior. p. 271-288. In: Huxley, C.R. \& D.F. Cuttler (Eds.) Ant-plant Interactions. New York, Oxford University Press, 601p.

Longino, J.T., 1996. Taxonomic characterization of some livestem inhabiting Azteca (Hymenoptera: Formicidae) in Costa Rica, with special reference to the ants of Cordia (Boraginaceae) and Triplaris (Polygonaceae). Journal of Hymenoptera Research, 5: 131-156.

Lorenzi, H., 2002. Árvores Brasileiras: Manual de identificação e cultivo de plantas arbóreas nativas do Brasil. Nova Odessa, Instituto Plantarum de Estudos da Flora LTDA. v. 2, 2 edição, $368 \mathrm{p}$.

Ness, J., K. Mooney \& L. Lach, 2009. Ants as Mutualists, p. 97114. In: Lach, L., C.L. Parr \& K.L. Abbott (Eds.) Ant Ecology. New York, Oxford University Press, 432p.

Putz, F.E. \& N.M. Holbrook, 1988. Further observations on the dissolution of mutualism between Cecropia and its ants: the Malaysian case. Oikos, 53: 121-125.

Rickson, F.R., 1977. Progressive loss of ant-related traits of Cecropia peltata on selected Caribbean Islands. American Journal of Botany, 64: 585-592.

Rico-Gray, V. \& P.S. Oliveira, 2007. The ecology and evolution of ant-plants interactions. Chicago, University of Chicago Press, 331p.

Sagers, C.L., S.M. Ginger \& R.D. Evans, 2000. Carbon and nitrogen isotopes trace nutrient exchange in an ant-plant mutualism. Oecologia, 123: 582-586.

Schultz, T.R. \& T.P. McGlynn, 2000. The interactions of ants with other organisms, p. 35-44. In: Agosti, D., J.D. Majer,
L.E. Alonso \& T.R. Schultz (Eds.) Ants: standard methods for measuring and monitoring biodiversity. Washington, Smithsonian Institution Press, 28op.

Schupp, E.W., 1986.Azteca protection of Cecropia: ant occupation benefits juvenile trees. Oecologia, 70: 379-385.

Val, E.D. \& R. Dirzo, 2003. Does ontogeny cause changes in the defensive strategies of the myrmecophyte Cecropia peltata? Plant Ecology, 169: 35-41.

Vasconcelos, H.L. \& A.B. Casimiro, 1997. Influence of Azteca alfari ants on the exploitation of Cecropia trees by a leafcutting ant. Biotropica, 29: 84-92.

Wetterer, J.K., 1997. Ants on Cecropia in Hawaii. Biotropica, 29: 128-132.

Wheeler, W.M., 1942. Studies of Neotropical ant-plants and their ants. Bulletin of the Museum of Comparative Zoology N. 1.

White, P.S., 1983. Corner's rules in eastern deciduous trees: allometry and its implications for the adaptive architecture of trees. Bulletin of the Torrey Botanical Club, 110: 203-212.

Yu, D.W. \& D.W. Davidson, 1997. Experimental studies of species-specificity in Cecropia-ant relationships. Ecological Monographs, 67: 273-294

\section{Recebido em: 24/10/2014 \\ Aceito em: 17/o6/2015}

\section{Como citar este artigo:}

Oliveira, G.V. de, M.M. Correa \& J.H. Delabie, 2015. Interações Planta-Inseto: Aspectos Biológicos e Ecológicos do Mutualismo Cecropia-Azteca. EntomoBrasilis, 8 (2): 85-90.

Acessível em: doi:10.12741/ebrasilis.v8i2.486
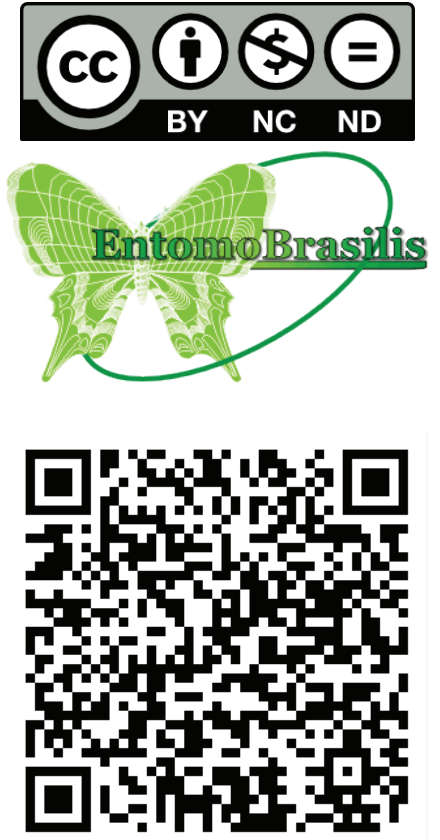